\title{
Dielectric Anomaly and Charge Fluctuations in the Non-Magnetic Dimer Mott Insulator $\lambda$-(BEDT-STF $)_{2} \mathrm{GaCl}_{4}$
}

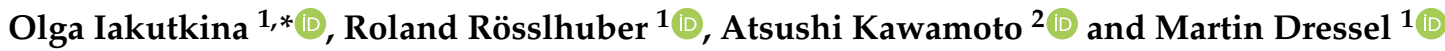 \\ 1 1. Physikalisches Institute, Universität Stuttgart, 70569 Stuttgart, Germany; \\ roland.roesslhuber@pi1.physik.uni-stuttgart.de (R.R.); dressel@pi1.physik.uni-stuttgart.de (M.D.) \\ 2 Department of Physics, Graduate School of Science, Hokkaido University, Sapporo 060-0810, Japan; \\ atkawa@phys.sci.hokudai.ac.jp \\ * Correspondence: olga.iakutkina@pi1.uni-stuttgart.de
}

Citation: Iakutkina, O.; Rösslhuber,

R.; Kawamoto, A.; Dressel, M.

Dielectric Anomaly and Charge

Fluctuations in the Non-Magnetic

Dimer Mott Insulator

$\lambda$-(BEDT-STF $)_{2} \mathrm{GaCl}_{4}$. Crystals 2021,

11, 1031. https://doi.org/

10.3390/cryst11091031

Academic Editor: Ingo Dierking

Received: 20 July 2021

Accepted: 24 August 2021

Published: 27 August 2021

Publisher's Note: MDPI stays neutral with regard to jurisdictional claims in published maps and institutional affiliations.

Copyright: (c) 2021 by the authors. Licensee MDPI, Basel, Switzerland. This article is an open access article distributed under the terms and conditions of the Creative Commons Attribution (CC BY) license (https:/ / creativecommons.org/licenses/by/ $4.0 /)$.

\begin{abstract}
The dimer Mott insulator $\lambda$-(BEDT-STF $)_{2} \mathrm{GaCl}_{4}$ undergoes no magnetic order down to the lowest temperatures, suggesting the formation of a novel quantum disordered state. Our frequency and temperature-dependent investigations of the dielectric response reveal a relaxor-like behavior below $T \approx 100 \mathrm{~K}$ for all three axes, similar to other spin liquid candidates. Optical measurement of the charge-sensitive vibrational mode $v_{27}\left(b_{1 u}\right)$ identifies a charge disproportionation $\Delta \rho \approx 0.04 e$ on the dimer that exists up to room temperature and originates from inequivalent molecules in the weakly coupled dimers. The linewidth of the charge sensitive mode is broader than that of typical organic conductors, supporting the existence of a disordered electronic state.
\end{abstract}

Keywords: strongly correlated systems; organic conductors; relaxor-ferroelectrics; dielectric spectroscopy; infrared spectroscopy; disordered systems

\section{Introduction}

Strongly correlated electron systems attract much interest due to some novel magnetic, dielectric, and superconducting properties; both electron-electron interactions and quantum fluctuations are considered crucial for understanding these phenomena. Among these systems, the quasi-two-dimensional organic charge-transfer salts are renowned for their versatility and enormously rich phase diagrams, comprising superconducting, spin-liquid, antiferromagnetic, or charge-ordered phases that arise from the interplay of spin, charge, and lattice degrees of freedom [1-5]. Besides the most popular examples $\kappa$-(BEDT-TTF $)_{2} X$, another dimerized family has drawn large attention, the $\lambda$-salts, where the lattice system consists of triangular and square tiling as depicted in Figure $1 \mathrm{~b}$. In addition to the well-studied unconventional superconducting properties, such as a Fulde-Ferrell-LarkinOvchinnikov state and field-induced superconductivity at strong magnetic fields [6-9], a spin-liquid-like state was discovered recently $[10,11]$.

The electronic phase of the insulating $\lambda$-(BEDT-STF $)_{2} \mathrm{GaCl}_{4}$ is situated between the antiferromagnet $\lambda$-(BEDT-TTF) ${ }_{2} \mathrm{GaCl}_{4}$ and the superconductor $\lambda$-(BETS) ${ }_{2} \mathrm{GaCl}_{4}$ (cf. Figure $1 \mathrm{a}$ for the molecular structure). While the most insulting compound $\lambda$-(BEDT-TTF) ${ }_{2} \mathrm{GaCl}_{4}$ undergoes an antiferromagnetic transition at $T_{N}=13 \mathrm{~K}$, no magnetic order occurs in $\lambda$-(BEDT-STF) ${ }_{2} \mathrm{GaCl}_{4}$ down to $1.63 \mathrm{~K}$ regardless of the strong coupling $J=194 \mathrm{~K}$ [12]. The temperature dependence of the magnetic susceptibility can be described by a $S=\frac{1}{2}$ two-dimensional antiferromagnetic Heisenberg model on the triangular lattice, suggesting geometrically frustrated spin-liquid-like behavior. However, a nuclear magnetic resonance (NMR) study found an inhomogeneous electronic state; after an increase of $1 / T_{1}$, the NMR relaxation rate saturates at a low temperature, which is in stark contrast to the magnetic properties of other spin liquid candidates. Hence, $\lambda$-(BEDT-STF) ${ }_{2} \mathrm{GaCl}_{4}$ is considered a realization of a novel quantum disordered state [11]. 
(a)<smiles></smiles><smiles></smiles><smiles>[B]C(F)(F)C1CSC2=C(S1)[Se]C(=C1[Se]C3=C(SCCS3)[Se]1)[Se]2</smiles>

(b)

$\kappa$

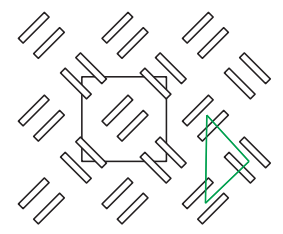

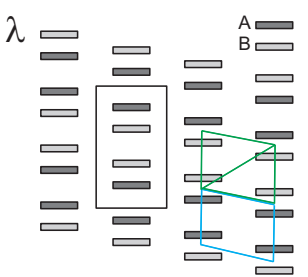

Figure 1. (a) The donor molecule ET = BEDT-TTF, i.e., bis-(ethylenedithio)-tetrathiafulvalene), is the most common building block, but sulfur can be replaced by selenium, leading to STF = BEDT-STF, i.e., bis-(ethylenedithio)-diseleniumdithiafulvalene, and BETS = BEDT-TSF, i.e., bis-(ethylenedithio)tetraselenafulvalene. The more extended orbitals cause a larger bandwidth favoring better conductivity. (b) The two-dimensional charge transfer salts form different dimer patterns, where two crystallographically independent donors crystallize face-to-face. In the $\kappa$-phase the dimers are rotated with respect to each other, while the $\lambda$-pattern is organized in stacks with two dimers per unit cell; here, the constituent molecules $\mathrm{A}$ and $\mathrm{B}$ differ by symmetry. The unit cell given by black contains four molecules $(\mathrm{A}, \mathrm{B})$ and $\left(\mathrm{B}^{\prime}, \mathrm{A}^{\prime}\right)$. The triangular arrangement of the dimers is indicated in green, where-depending on the particular transfer integrals—a high degree of frustration can be reached. Due to the weaker diagonal interaction, a square tiling occurs, shown by blue lines.

Having in mind that numerous organic conductors including antiferromagnets and spin-liquid candidates exhibit dielectric anomalies [13-24], with the charge-order driven ferroelectric state detected for some of them [25-27], and the growing numbers of possible applications of ferroelectric materials [28-32], understanding the mechanism of dielectric anomaly and investigating the charge dynamics of the electronic state in organic conductors are of great interest. In this study, we focused on the disordered quantum state of $\lambda$ type salts.

To this end, we employ dielectric and vibration spectroscopies to explore the charge state and the presence of the dielectric anomaly in $\lambda$-(BEDT-STF $)_{2} \mathrm{GaCl}_{4}$; in addition, the compound is investigated by infrared spectroscopy as the standard and very powerful tool to elucidate the charge distribution on the molecules [33-38].

\section{Experimental Details}

Single crystals of $\lambda$-(BEDT-STF $)_{2} \mathrm{GaCl}_{4}$ were synthesized at Hokkaido University by the standard electrochemical oxidation method [39]. In contrast to BEDT-TTF molecules, in BEDT-STF, two central sulfur atoms are substituted by Se atoms, leading to asymmetric BEDT-STF molecules as sketched in Figure 1a. The crystals have a needle-like shape parallel to the $c$-axis and typical dimensions of $1 \mathrm{~mm} \times 0.2 \mathrm{~mm} \times 0.05 \mathrm{~mm}$. The donor molecules are dimerized with the pairs arranged in the ac-plane. As shown in Figure 2, the conducting layers of donor molecules alternate with insulating anion sheets along the $b$-axis, giving rise to a quasi-two-dimensional structure. The morphology of the crystals corresponds to the (11̄0), (110), and (001)-planes. For clarity (11̄0) and (110) planes are depicted in Figure 2c, while the (001)-plane coincides with the c-axis. 
(a)

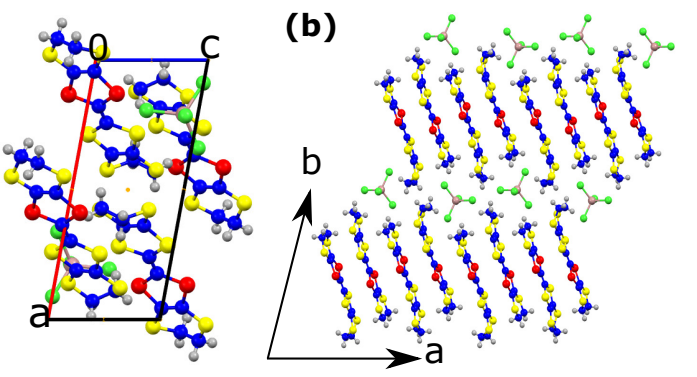

(c)

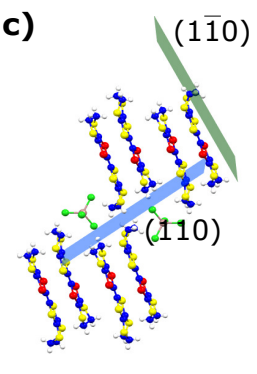

Figure 2. Crystal structure of $\lambda$-(BEDT-STF) ${ }_{2} \mathrm{GaCl}_{4}$. (a) The $\lambda$-type arrangement of donor molecules within the highly conducting ac-plane. (b) The layered structure becomes obvious when looking along the $c$-direction, where the alternation of donor and anion layers are seen. (c) Green and blue planes correspond to (110) and (110), respectively.

Dielectric measurements between $T=295$ and $7 \mathrm{~K}$ are carried out using an Agilent A4294 impedance analyzer that covers the frequency range $100 \mathrm{~Hz}-10 \mathrm{MHz}$. The spectra of the complex dielectric permittivity

$$
\hat{\epsilon}(\omega)=\frac{\hat{\sigma}(\omega)-\sigma_{0}}{\mathrm{i} \epsilon_{0} \omega}=\epsilon^{\prime}(\omega)+\mathrm{i} \epsilon^{\prime \prime}(\omega)
$$

are obtained along all three directions, [110], [110], and [001], using the two-contact method, where gold wires are attached on both sides of the crystal with carbon paste; the other ends of the wires are connected to the sample holder with silver paint. Here, $\sigma_{0}$ is the conductivity caused by free electrons in the material, and $\epsilon_{0}$ is the vacuum permittivity; $\epsilon^{\prime}$ and $\epsilon^{\prime \prime}$ are the real and imaginary parts of the permittivity. To have reliable data, the sample holder open-loop contribution is subtracted [40]. Using a continuous helium-flow cryostat (KONTI by CryoVac, Troisdorf, Germany), the samples can be cooled down from room temperature to $T=7 \mathrm{~K}$.

Optical reflectivity measurements off the (110)-plane of $\lambda$-(BEDT-STF) ${ }_{2} \mathrm{GaCl}_{4}$ single crystals are carried out with a Bruker Hyperion infrared microscope attached to a Bruker Vertex 80v Fourier-transform infrared spectrometer. The experiments are performed with the light polarized parallel to $[1 \overline{1} 0]$, i.e., in the direction most sensitive to the chargesensitive infrared-active intramolecular vibrational mode $v_{27}\left(b_{1 u}\right)[35,41,42]$. The spectra are recorded in a frequency range from 500 to $8000 \mathrm{~cm}^{-1}$ between $T=295$ and $12 \mathrm{~K}$. The optical conductivity is calculated via the Kramers-Kronig transformation with constant extrapolation of reflectivity below $500 \mathrm{~cm}^{-1}$, which is common for insulators, and using standard $\omega^{-4}$ decay as high-frequency extrapolation.

\section{Results and Analysis}

\subsection{Dielectric Properties}

Figure 3 displays the real part of the dielectric permittivity $\epsilon^{\prime}(T)$ as a function of temperature measured at various frequencies $f=\omega / 2 \pi$ along the three directions [001], $[110]$, and $[1 \overline{1} 0]$ of a $\lambda$-(BEDT-STF $)_{2} \mathrm{GaCl}_{4}$ crystal.

For the orientations $E \|[001]$ and $E \|[1 \overline{1} 0]$, broad maxima develop below $T=100 \mathrm{~K}$, which are strongly frequency dependent. With decreasing frequency, the peak shifts toward lower temperatures and becomes sharper. For $E \|[110]$, a clear step can be seen in the real part of the dielectric constant around $T=60 \mathrm{~K}$ that shifts toward higher temperatures with increasing frequency. This behavior is typical for relaxor ferroelectrics, and similar dielectric anomalies are frequently observed in organic conductors [19-21,43,44]. 


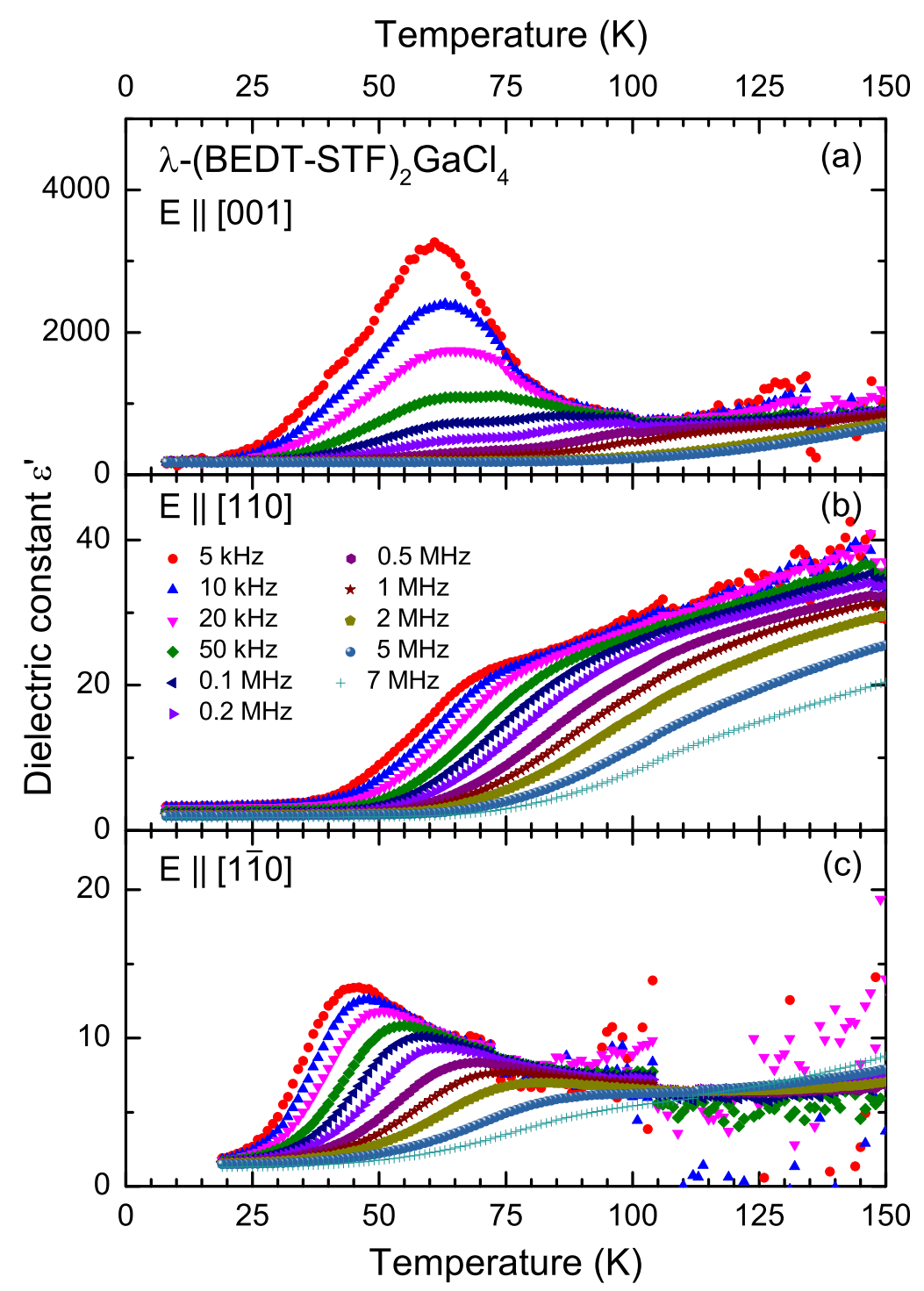

Figure 3. Temperature-dependent real part of the permittivity of $\lambda$-(BEDT-STF $)_{2} \mathrm{GaCl}_{4}$ recorded at different frequencies for the electric field along the three different directions, i.e., (a) $E \|[001]$, (b) $E \|[110]$, and (c) $E \|[1 \overline{1} 0]$.

Dielectric relaxation appears in a rather broad temperature range; for intermediate temperatures, the frequency dependence of the real and imaginary parts of the dielectric permittivity is plotted in Figure 4 . The overall behavior can be described by the generalized Debye model $[45,46]$ :

$$
\hat{\epsilon}(\omega)-\epsilon_{\infty}=\frac{\Delta \epsilon}{1+\left(i \omega \tau_{o}\right)^{1-\alpha}}
$$

where $\Delta \epsilon=\epsilon^{\prime}(\omega \rightarrow 0)-\epsilon^{\prime}(\omega \rightarrow \infty)$ is a measure of the dielectric strength, and $\epsilon^{\prime}(\omega \rightarrow 0)$ and $\epsilon^{\prime}(\omega \rightarrow \infty)$ are the limiting static and the high-frequency values of the dielectric constant, respectively. $\tau_{0}$ is the mean relaxation time, and $(1-\alpha)$ is the symmetric broadening. The drop in $\epsilon^{\prime}(\omega)$ with increasing frequency implies that the dipoles cannot follow the ac electric field at high frequencies [47]. Since $\epsilon^{\prime}(\omega)$ and $\epsilon^{\prime \prime}(\omega)$ are linked via the KramersKronig relation, the step in the real part results in a peak in the absorption $\epsilon^{\prime \prime}$. The solid black lines in Figure 4 represent the fit of the data according to Equation (2), and the dashed green line is the dc contribution $\sigma_{0}$.

The temperature dependence of the parameters of $\lambda$-(BEDT-STF) ${ }_{2} \mathrm{GaCl}_{4}$ extracted from the fit of the data by Equation (2) is plotted in Figure 5 as a function of inverse 
temperature $1 / T$. The dielectric strength $\Delta \epsilon$ exhibits a broad peak around $T=67 \mathrm{~K}$ for the electric field oriented within the (11̄0)-plane and around $T=40 \mathrm{~K}$ for the out of plane direction, $E \|[1 \overline{1} 0]$. This behavior resembles the temperature dependence of $\epsilon^{\prime}(T)$ for low frequencies. At high temperatures, the mean relaxation time $\tau_{0}(T)$ shows thermally activated behavior for all three directions; at the same time, the symmetric broadening $(1-\alpha)$ decreases with decreasing temperature. These features are signatures of cooperative behavior and glass-like freezing of molecular motion [48].

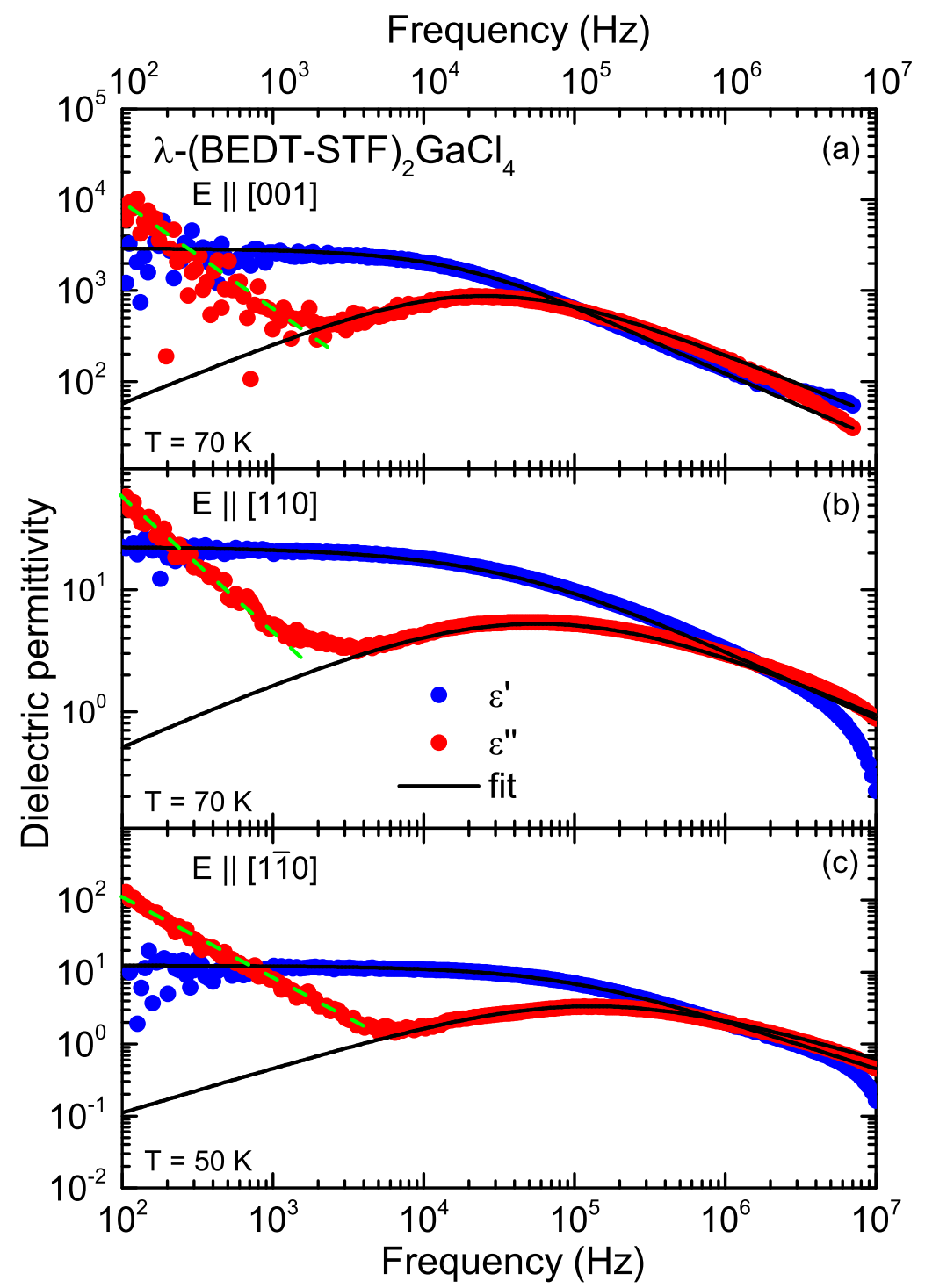

Figure 4. Double logarithmic presentation of the frequency-dependent real and imaginary parts of the dielectric permittivity of $\lambda$-(BEDT-STF) ${ }_{2} \mathrm{GaCl}_{4}, \epsilon^{\prime}$ (blue symbols) and $\epsilon^{\prime \prime}$ (red symbols). The data are recorded along (a) the [001]- and (b) [110]-directions at $T=70 \mathrm{~K}$ and (c) for $E \|[1 \overline{1} 0]$ at $T=50 \mathrm{~K}$. The solid black lines represent fits by the generalized Debye model; the dashed green lines indicate the dc contribution to the imaginary part of the permittivity.

The temperature dependence of the mean relaxation time for disordered glassy systems with critical slowing down is commonly described by the Vogel-Fulcher-Tammann (VFT) expression [48,49]:

$$
\tau_{0}(T)=\tau_{\mathrm{VFT}} \exp \left\{\frac{\Delta_{\mathrm{VFT}}}{T-T_{\mathrm{VFT}}}\right\},
$$


where $\Delta_{\mathrm{VFT}}$ is the activation energy for reorientational motion, $T_{\mathrm{VFT}}$ is the temperature where the mean relaxation time diverges, and $\tau_{\mathrm{VFT}}$ is the time scale for the response in the high-temperature limit.

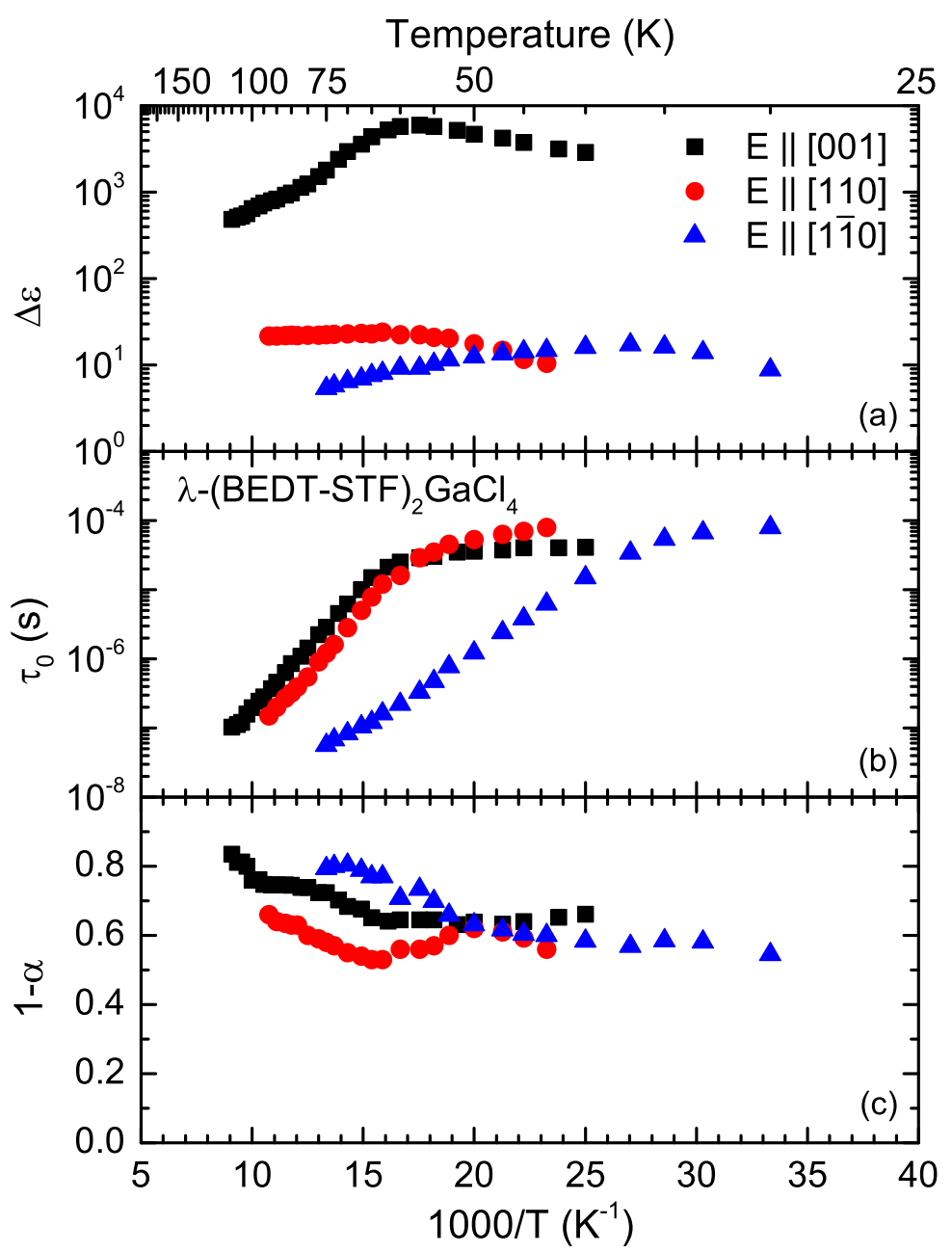

Figure 5. (a) Dielectric strength $\Delta \epsilon(T),(\mathbf{b})$ mean relaxation time $\tau_{0}(T)$, and (c) symmetric broadening $(1-\alpha)$ as a function of inverse temperature for all the directions of $\lambda$-(BEDT-STF) ${ }_{2} \mathrm{GaCl}_{4}$, as indicated.

In Figure 6 , the mean relaxation time $\tau_{0}(T)$ of $\lambda$-(BEDT-STF $)_{2} \mathrm{GaCl}_{4}$ is plotted for all three orientations as a function of $1 /\left(T-T_{V F T}\right)$ together with fits by Equation (3). The Vogel-Fulcher-Tammann law explains how the peak seen in the temperature-dependent plot $\epsilon^{\prime}(T)$ of Figure 3 shifts with frequency. The parameters of the mean relaxation time obtained by the fits are listed in Table 1 for the different axes. We also see that $T_{\mathrm{VFT}}$ is equal to $15 \mathrm{~K}$ for all three directions; the value is slightly higher than $T_{\mathrm{VFT}} \approx 6 \mathrm{~K}$, extracted for the spin liquid candidate $\kappa$-(BEDT-TTF $)_{2} \mathrm{Cu}_{2}(\mathrm{CN})_{3}$, adjusted to the anomaly observed in numerous other quantities [44].

Table 1. The Vogel-Fulcher-Tammann parameters of $\lambda$-(BEDT-STF) $)_{2} \mathrm{GaCl}_{4}$ : the mean relaxation time $\tau_{0}$, activation energy $\Delta_{\mathrm{VFT}}$, and glass temperature $T_{\mathrm{VFT}}$ obtained for $E\|[001], E\|[110]$, and $E \|[1 \overline{1} 0]$.

\begin{tabular}{rccc}
\hline $\begin{array}{r}\text { Directions of } \\
\text { Measurement }\end{array}$ & $\begin{array}{c}\tau_{\text {VFT }} \\
(\mathbf{s})\end{array}$ & $\begin{array}{c}\Delta_{\text {VFT }} \\
(\mathbf{K})\end{array}$ & $\begin{array}{c}T_{\text {VFT }} \\
(\mathbf{K})\end{array}$ \\
\hline$[001]$-axis & $2.5 \times 10^{-10}$ & 560 & 15 \\
{$[110]$-axis } & $1.85 \times 10^{-10}$ & 525 & 15 \\
{$[1 \overline{1} 0]$-axis } & $8.2 \times 10^{-10}$ & 250 & 15 \\
\hline
\end{tabular}


The anisotropy of the activation energy extracted in $\kappa$-(BEDT-TTF $)_{2} \mathrm{Cu}_{2}(\mathrm{CN})_{3}$ also ranged up to a factor of 2 in the Vogel-Fulcher-Tammann fit with remarkable deviations between different single crystals, reaching up to $510 \mathrm{~K}$ and $330 \mathrm{~K}$, respectively $[19,20]$. Hence, from the slowing down of the relaxation time according to an Arrhenius behavior, Pinterić et al. obtain values comparable to the ones given in Table 1 with a glass temperature between 10 and $15 \mathrm{~K}$. Again, the sample-to-sample deviation indicates disorder being important for $\kappa$-(BEDT-TTF $)_{2} \mathrm{Cu}_{2}(\mathrm{CN})_{3}$ and also for $\kappa$-(BEDT-TTF $)_{2} \mathrm{Ag}_{2}(\mathrm{CN})_{3}$ [20-22]

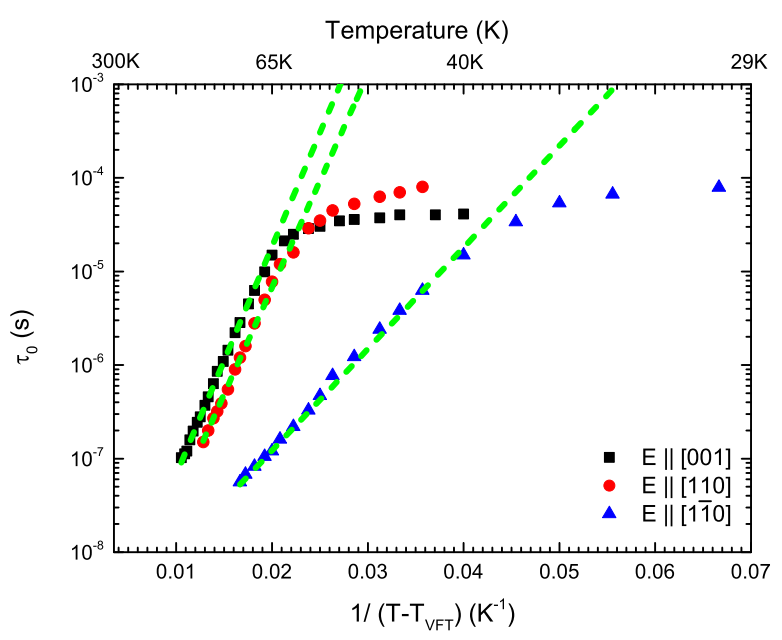

Figure 6. Arrhenius presentation of the mean relaxation time $\tau_{0}(T)$ of $\lambda$-(BEDT-STF $)_{2} \mathrm{GaCl}_{4}$ measured along the [001] (black square), [110] (red circle), and [110] (blue triangle) axes. The solid green line corresponds to fits by the Vogel-Fulcher-Tammann expression (3).

Alternatively, the temperature dependence of the real part of the dielectric permittivity displayed in Figure 3 might be described by a Curie-Weiss behavior. The Curie-Weiss law for the static dielectric constant as a function of temperature has the form

$$
\epsilon^{\prime}(T)=\frac{C}{T-T_{C}}
$$

where $C$ is the Curie constant, and $T_{C}$ is the Curie temperature. In Figure 7, we plot $\epsilon^{\prime}(T)$ along $E \|[001]$ and $E \|[1 \overline{1} 0]$ for several frequencies.

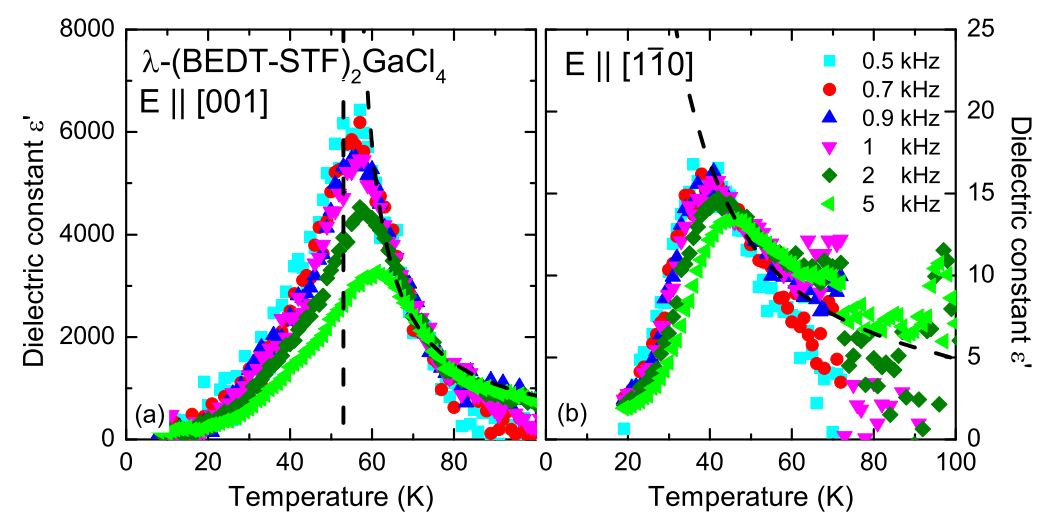

Figure 7. The real part of the dielectric constant $\epsilon^{\prime}(T)$ plotted as a function of temperature at certain frequencies for (a) $E \|[001]$ and (b) $E \|[1 \overline{1} 0]$. The dashed black line corresponds to the Curie-Weiss fit according to Equation (4).

The best fit by Equation (4) for frequencies less than $5 \mathrm{kHz}$ is given by the dashed line; no clear Curie-Weiss peak is visible for the direction $E \|[110]$. The obtained parameters are listed in Table 2. From the Curie constant $C$ for $E \|[1 \overline{1} 0]$ (out of plane), we can estimate 
the dipole strength following the procedure described by Pinterić et al. [20]. Assuming that the dielectric behavior is a result of charge imbalance within the dimers, we can estimate the amount of charge disproportionation $\Delta \rho \approx 0.05 e$.

Table 2. Parameters $C$ and $T_{C}$ of $\lambda$-(BEDT-STF $)_{2} \mathrm{GaCl}_{4}$ obtained from the fit of $\epsilon^{\prime}(T)$ by the CurieWeiss Equation (4) for $E \|[001]$ and $E \|[1 \overline{1} 0]$.

\begin{tabular}{rcc}
\hline $\begin{array}{r}\text { Directions of } \\
\text { Measurement }\end{array}$ & $\begin{array}{c}\boldsymbol{C} \\
(\mathbf{K})\end{array}$ & $\begin{array}{c}\boldsymbol{T}_{\boldsymbol{C}} \\
(\mathbf{K})\end{array}$ \\
\hline$[001]$-axis & $4 \times 10^{4}$ & 53 \\
{$[1 \overline{1} 0]$-axis } & 420 & 15 \\
\hline
\end{tabular}

\subsection{Vibrational Spectroscopy}

In order to learn about the possible charge disproportionation in $\lambda$-(BEDT-STF) ${ }_{2} \mathrm{GaCl}_{4}$ on a microscopic scale, we apply infrared spectroscopy frequently used for investigating organic charge-transfer salts [33-38,41,50]. Here, we focus on the asymmetric infraredactive vibrational mode $v_{27}\left(\mathrm{~b}_{1 u}\right)$ that involves the $\mathrm{C}=\mathrm{C}$ bonds and is thus very sensitive to the electronic charge per molecule. It can be probed best perpendicular to the plane, i.e., for $E \|[1 \overline{1} 0]$. Figure 8a displays the optical conductivity $\sigma_{1}(\omega)$ of $\lambda$-(BEDT-STF) ${ }_{2} \mathrm{GaCl}_{4}$ in the corresponding spectral range for several temperatures. The broad band observed around $1465 \mathrm{~cm}^{-1}$ is assigned to the $v_{27}$ vibration for half a hole per BEDT-TTF molecule. The feature becomes more pronounced upon cooling, but soon it becomes obvious that it is composed of two modes; eventually, two peaks are well separated.

For the quantitative characterization, the conductivity spectra of the $v_{27}$ mode can be described satisfactorily with one Fano function above $T=90 \mathrm{~K}$, while two Lorentzians are necessary below. For the optical conductivity, the Fano function and one Lorentzian give $\sigma_{1}^{\text {Fano }}$ and $\sigma_{1}^{\text {Lorentz }}$, respectively, as described in, e.g., Equation $(5 \mathrm{a}, \mathrm{b})$ :

$$
\begin{gathered}
\sigma_{1}^{\text {Fano }}(v)=\sigma_{0} \frac{\gamma v\left[\gamma v\left(q^{2}-1\right)+2 q\left(v^{2}-v_{0}^{2}\right)\right]}{\left(v^{2}-v_{0}^{2}\right)^{2}+\gamma^{2} v^{2}} \\
\sigma_{1}^{\text {Lorentz }}(v)=\frac{\sigma_{0} \gamma v^{2}}{\left(v^{2}-v_{0}^{2}\right)^{2}+\gamma^{2} v^{2}}
\end{gathered}
$$

where $v=f / c=\omega /(2 \pi c), \sigma_{0}$, and $\gamma$ are frequency, amplitude, and damping, respectively, and $q$ is a phenomenological coupling constant in the Fano function, which gives the Lorentzian shape in the case $q= \pm \infty$.

In Figure 8b,c, we present examples of the fits to $\sigma_{1}(\omega)$ at $T=295$ and $12 \mathrm{~K}$; in addition, a broad Lorentzian accounts for the electronic background. The peak frequencies and linewidths are plotted in panels (d) and (e) as a function of temperature. While some hardening is observed for $T>150 \mathrm{~K}$, the mode frequency saturates when cooling further. This also holds when we fit the spectra by two modes at low temperatures. Besides some thermal narrowing, when cooling starts at room temperature, the overall linewidth remains constant at approximately $13 \mathrm{~cm}^{-1}$ below $150 \mathrm{~K}$. 

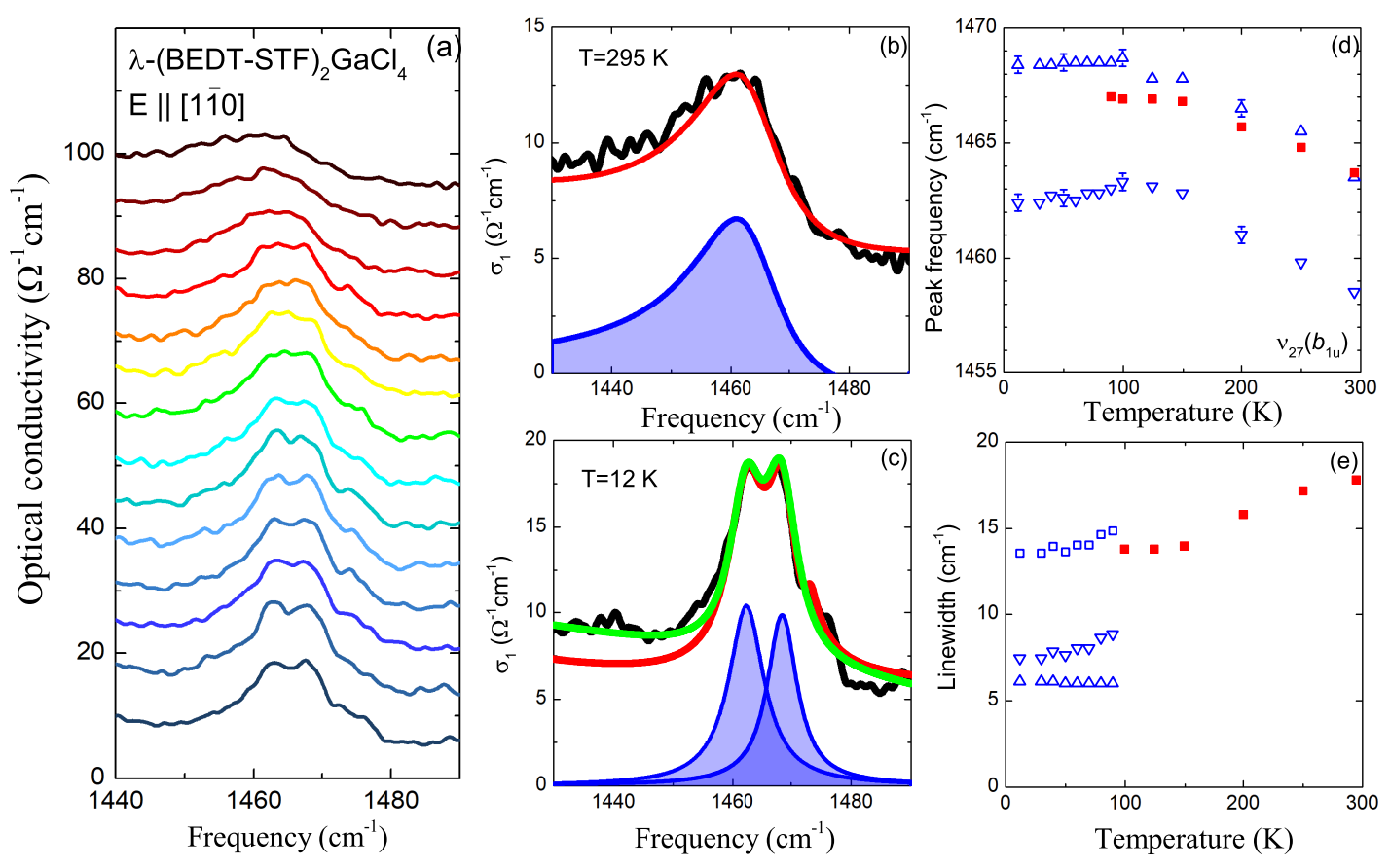

Figure 8. (a) Temperature evolution of the optical conductivity of $\lambda$-(BEDT-STF) ${ }_{2} \mathrm{GaCl}_{4}$ in the region of the molecular vibration $v_{27}\left(\mathrm{~b}_{1 u}\right)$. The data are shifted with respect to each other by a constant offset for clarity reasons. $(\mathbf{b}, \mathbf{c})$ Fits of the vibrational mode at $T=295$ and $12 \mathrm{~K}$. The experimental data are shown in black, the red lines correspond to the overall fit, and the blue lines are separate contributions to the mode. From the two-state jump model [Equation (7)], we obtain the green line. (d,e) Temperature dependence of the resonance frequency and linewidth of the charge-sensitive mode $v_{27}\left(\mathrm{~b}_{1 u}\right)$. While the red squares correspond to the fit by a single contribution for $T \geq 90 \mathrm{~K}$, the blue symbols represent the two-mode description, where triangles and rotated triangles are related to different Lorentzians; the open blue squares in panel (e) correspond to the sum of both, demonstrating that the overall width does not change.

In Figure $8 \mathrm{~d}$, the temperature-dependent results from fitting the $v_{27}\left(\mathrm{~b}_{1 u}\right)$ vibrational feature of $\lambda$-(BEDT-STF $)_{2} \mathrm{GaCl}_{4}$ by two Lorentzian modes are displayed by blue symbols. For $T<90 \mathrm{~K}$, the peaks are well separated, but we can also extend this approach to higher temperatures, as an alternative to the description by a single Fano-line (red squares). Obviously, there is a kink in the temperature evolution of the vibrational frequency around $T=100 \mathrm{~K}$ that is independent of the fit procedure. At elevated temperatures, the blue-shift upon cooling follows the typical thermal hardening. The kink in this behavior at around $100 \mathrm{~K}$ infers some modification in the physical properties. Even though the origin of this kink is unclear, it can be related to the realization of an inhomogeneous electronic state suggested from NMR measurements, where an increasing linewidth was observed in the same temperature range, and the temperature dependence of the dc resistivity follows variable-range hopping or soft Hubbard gap models below $100 \mathrm{~K}$, characteristic for systems with disorder $[51,52]$. It is also interesting to note that this anomaly occurs exactly at the temperature where the dielectric dispersion starts to develop, as shown in Figure 3.

Let us first have a look at this separation. The splitting of the $v_{27}\left(\mathrm{~b}_{1 u}\right)$ molecular vibration is commonly taken as evidence that there are two distinct molecules containing unequal charge, and the charge imbalance $\Delta \rho$ can be simply determined as the differences between charges on these two molecules, $\Delta \rho=\rho$ (charge rich) $-\rho$ (charge poor). From the separation of the two peaks by $\Delta v=6 \mathrm{~cm}^{-1}$ extracted from Figure $8 \mathrm{~d}$, we can estimate the charge imbalance $\Delta \rho$ according to

$$
\Delta v_{27}=-\left(140 \mathrm{~cm}^{-1} / e\right) \Delta \rho,
$$

suggested for BEDT-TTF compounds [35,41]; here we would like to note that, despite this relation was established for the BEDT-TTF molecules, it will also hold for BEDT-STF, as 
the replacement of some $S$ ions by Se in BEDT-STF or BETS leads to only a small shift in $v_{27}$ of less than 2 or $3 \mathrm{~cm}^{-1}$, respectively, in absolute value [53,54]. From our data we obtain $\Delta \rho \approx 0.043 e$ which is independent of temperature. Although the mode is thermally broadened at higher temperatures, the fit by two terms can be extended up to $T=300 \mathrm{~K}$ without change in the frequency separation. This implies that the charge disproportionation of $\Delta \rho$ is already present at an ambient condition and remains unaffected by temperature. In other words, there is no charge-order phase transition in $\lambda$-(BEDT-STF) ${ }_{2} \mathrm{GaCl}_{4}$, comparable to the one seen in one-dimensional charge transfer salts as a mean-field development of the charge disproportionation $[44,55]$.

The behavior is also distinct from $\kappa-(\mathrm{BEDT}-\mathrm{TTF})_{2} \mathrm{Hg}(\mathrm{SCN})_{2} \mathrm{Br}$, where a second peak develops around $18 \mathrm{~cm}^{-1}$ below the main peak, which, in fact, also exhibits a double structure with a sideband $5 \mathrm{~cm}^{-1}$ apart [24]. In the present case, we do not see a shoulder on one side gradually developing towards full peaks; instead, we observe a vibrational feature that is rather broad, and it becomes more pronounced upon cooling without strongly increasing or decreasing in width.

This observation is in line with the presence of two crystallographically inequivalent donor molecules, $\mathrm{A}$ and $\mathrm{B}$, forming the dimer in the $\lambda$-type salts, as depicted in Figure $1 \mathrm{~b}$. We conclude that these molecules are not only distinct by symmetry but also carry different charges. The charge imbalance is rather small when compared to the non-dimerized $\alpha$ $(\text { BEDT-TTF })_{2} \mathrm{I}_{3}$, for instance, where a charge disproportionation of more than $\Delta \rho \approx 0.1 e$ is already present at room temperature well above the charge-order transition [56]. To our knowledge, there are no systematic vibrational studies of the family of $\lambda$-salts. In the related compound $\lambda$-(BETS) ${ }_{2} \mathrm{GaCl}_{4}$, a weak charge disproportionation was concluded from the broadening of the ${ }^{77}$ Se NMR spectrum and its angular dependence [57].

Most dimerized charge-transfer systems, such as $\kappa-(B E D T-T T F)_{2} \mathrm{Cu}_{2}(\mathrm{CN})_{3}$ or $\kappa-(\mathrm{BEDT}-$ $-\mathrm{TTF})_{2} \mathrm{Cu}\left[\mathrm{N}(\mathrm{CN})_{2}\right] \mathrm{Cl}$, do not develop any charge disproportionation beyond $1 \%$, which is about the experimental resolution [58]. However, with approximately $6.5 \mathrm{~cm}^{-1}$, the vibrational features of $\kappa-(\mathrm{BEDT}-\mathrm{TTF})_{2} \mathrm{Cu}_{2}(\mathrm{CN})_{3}$ are significantly broader than what is observed in typical charge-ordered compounds, such as $\alpha-(\mathrm{BEDT}-\mathrm{TTF})_{2} \mathrm{I}_{3}$, where the linewidth is less than $3 \mathrm{~cm}^{-1}[59,60]$, or in $\kappa-(\mathrm{BEDT}-\mathrm{TTF})_{2} \mathrm{Hg}(\mathrm{SCN})_{2} \mathrm{Cl}$ where the individual width is around 4 to $5 \mathrm{~cm}^{-1}$ at $T=10 \mathrm{~K}$ [24]. This was explained by intradimer charge fluctuations, using a two-state jump model [36]. A similar conclusion can be drawn from investigations of the Raman-active fully symmetric vibrations, $v_{2}$ and $v_{3}$ [61].

As seen from Figure $8 \mathrm{e}$, for $\lambda$-(BEDT-STF $)_{2} \mathrm{GaCl}_{4}$ the width of the $v_{27}$ modes also decreases only slightly with a reduced temperature and remains at about $13 \mathrm{~cm}^{-1}$ in total. If we assume an electronic charge fluctuating within the dimer, depending on the fluctuation rate, the broadening or splitting of the mode can be described by the Kubo formula [36]:

$$
\mathcal{L}(\omega)=\frac{\mathcal{F}\left[\left(\gamma+2 v_{\mathrm{ex}}\right)-\mathrm{i}\left(\omega-\omega_{\mathrm{w}}\right)\right]}{\mathcal{R}^{2}-\left(\omega-\omega_{1}\right)\left(\omega-\omega_{2}\right)-2 \mathrm{i} \Gamma\left(\omega-\omega_{\mathrm{av}}\right)} .
$$

Here, $\mathcal{F}=f_{1}+f_{2}$, with $f_{1}, f_{2}$ being the oscillator strengths of the bands at frequency $\omega_{1}$ and $\omega_{2}$ and halfwidth $\gamma$. The charge fluctuation velocity is $v_{\text {ex }}, \Gamma=\gamma+v_{\text {ex }}$ is the resulting width, and the abbreviation $\mathcal{R}^{2}=2 \gamma v_{\text {ex }}+\gamma^{2}$. Finally, we define the average and weighted frequency, $\omega_{\mathrm{av}}$ and $\omega_{\mathrm{w}}$, by

$$
\omega_{\mathrm{av}}=\frac{\omega_{1}+\omega_{2}}{2} \quad \text { and } \quad \omega_{\mathrm{w}}=\frac{f_{2} \omega_{1}+f_{1} \omega_{2}}{f_{1}+f_{2}} .
$$

When the charge oscillations are slow, $v_{\mathrm{ex}} \ll\left|\omega_{1}-\omega_{2}\right| / 2$, Equation (7) yields two separated bands centered around $\omega_{1}$ and $\omega_{2}$, while for $v_{\mathrm{ex}} \gg\left|\omega_{1}-\omega_{2}\right| / 2$, the motional narrowing will give one single band centered at the intermediate frequency $\omega_{\mathrm{av}}$. Finally, when $v_{\mathrm{ex}} \approx\left|\omega_{1}-\omega_{2}\right| / 2$, we shall observe one broad band shifted towards the mode with larger oscillator strength.

The green line in Figure 8c represents the fit of the data by Equation (7), with a slitting of $6.2 \mathrm{~cm}^{-1}$ and a fluctuation rate $v_{\mathrm{ex}}=0.3 \mathrm{~cm}^{-1}$ corresponding to $9 \times 10^{10} \mathrm{~s}^{-1}$. This 
exchange frequency is certainly slower than estimated for $\kappa-(\mathrm{BEDT}-\mathrm{TTF})_{2} \mathrm{Cu}_{2}(\mathrm{CN})_{3}$ but much faster than the $v_{\mathrm{ex}}=40 \mathrm{~cm}^{-1}$ obtained from Raman measurements on $\kappa$-(BEDT$\mathrm{TTF})_{2} \mathrm{Hg}(\mathrm{SCN})_{2} \mathrm{Cl}[62]$.

Although the estimated charge disproportionation of $\Delta \rho \approx 0.043 e$ is in good agreement with the value obtained from our dielectric measurements, we should keep in mind that this sort of charge fluctuation is much too fast to be the sole cause for the dielectric response observed in the $\mathrm{kHz}$ and $\mathrm{MHz}$ range of frequency. In addition, we do not observe any significant temperature dependence of the charge disproportionation among the molecules, which could be related to the significant temperature dependence of the dielectric behavior. The important facts in $\lambda$-(BEDT-STF $)_{2} \mathrm{GaCl}_{4}$ are the intrinsic disorder due to the asymmetric BEDT-STF molecules and the domain wall formation due to charge order, as discussed previously $[44,59,60]$. The random orientation of the asymmetric BEDT-STF molecules introduces inhomogeneous charge localization, giving rise to enhanced linewidth. Hence, it is more plausible that the disordered donor molecule structure plays a role for the broad linewidth as it provides a different chemical environment, suggesting that charge fluctuations are not dominant in the insulating phase next to the SC phase in the $\lambda$-salts. This supports NMR studies claiming that magnetic fluctuation should contribute to the SC pairing mechanism $[11,63]$. To check the effect of the charge fluctuation in detail, ultrasonic measurements will be useful. Of course, Raman scattering experiments should eventually be performed to verify our findings.

\section{Conclusions}

Dielectric and vibrational spectroscopies were performed on $\lambda$-(BEDT-STF $)_{2} \mathrm{GaCl}_{4}$ in order to elucidate the charge degrees of freedom. Our temperature and frequencydependent investigations of the dielectric properties reveal relaxor-like ferroelectric behavior below $T \approx 100 \mathrm{~K}$. The vibration spectroscopy found two $v_{27}$ modes which can be related to inequivalent donor molecules. The amount of charge disproportionation is consistently estimated to be approximately $\Delta \rho=0.04-0.05 e$, which remains temperature independent, ruling out a charge-order transition. At this point, we cannot give a final answer as to what causes the kink in the vibrational properties around $T=100 \mathrm{~K}$ and the concomitant occurrence of the anomaly in the dielectric constant. The linewidth of the $v_{27}$ mode is broader than that of typical BEDT-TTF salts, indicating that the asymmetric BEDT-STF molecules constitute a different chemical environment. This supports that the electronic state in $\lambda$-(BEDT-STF $)_{2} \mathrm{GaCl}_{4}$ is strongly influenced by disorder, leading to some novel quantum state, as previously suggested.

Author Contributions: Conceptualization, M.D. and O.I.; measurements and analysis, O.I. and R.R.; resources, M.D.; manuscript preparation, O.I. and M.D.; sample preparation: A.K. All authors have read and agreed to the published version of the manuscript.

Funding: This research was funded by by the DeutscheForschungsgemeinschaft (DFG) via DR228/39-3.

Institutional Review Board Statement: Not applicable.

Informed Consent Statement: Not applicable.

Data Availability Statement: Data are available from O.I. upon request.

Acknowledgments: We thank Gabriele Untereiner for the crucial technical support and Yohei Saito for fruitful discussions.

Conflicts of Interest: The authors declare no conflict of interest.

\section{References}

1. Lebed, A.G. The Physics of Organic Superconductors and Conductors; Springer Series in Materials Science; Springer: Berlin/Heidelberg, Germany, 2008; Volume 110.

2. Dressel, M. Quantum criticality in organic conductors? Fermi liquid versus non-Fermi-liquid behaviour. J. Phys. Condens. Matter 2011, 23, 293201. [CrossRef] 
3. Dressel, M.; Tomić, S. Molecular quantum materials: Electronic phases and charge dynamics in two-dimensional organic solids. Adv. Phys. 2020, 69, 1-120. [CrossRef]

4. Powell, B.J.; McKenzie, R.H. Quantum Frustration in Organic Mott Insulators: From Spin Liquids to Unconventional Superconductors. Rep. Progr. Phys. 2011, 74, 056501. [CrossRef]

5. Kurosaki, Y.; Shimizu, Y.; Miyagawa, K.; Kanoda, K.; Saito, G. Mott Transition from a Spin Liquid to a Fermi Liquid in the Spin-Frustrated Organic Conductor $\kappa-(\mathrm{ET})_{2} \mathrm{Cu}_{2}(\mathrm{CN})_{3}$. Phys. Rev. Lett. 2005, 95, 177001. [CrossRef] [PubMed]

6. Uji, S.; Terashima, T.; Nishimura, M.; Takahide, Y.; Konoike, T.; Enomoto, K.; Cui, H.; Kobayashi, H.; Kobayashi, A.; Tanaka, H.; et al. Vortex dynamics and the Fulde-Ferrell-Larkin-Ovchinnikov state in a magnetic-field-induced organic superconductor. Phys. Rev. Lett. 2006, 97, 157001. [CrossRef]

7. Uji, S.; Brooks, J.S. Magnetic-Field-Induced Superconductivity in Organic Conductors. J. Phys. Soc. Jpn. 2006, 75, 051014. [CrossRef]

8. Uji, S.; Shinagawa, H.; Terashima, T.; Yakabe, T.; Terai, Y.; Tokumoto, M.; Kobayashi, A.; Tanaka, H.; Kobayashi, H. Magneticfield-induced superconductivity in a two-dimensional organic conductor. Nature 2001, 410, 908. [CrossRef]

9. Ardavan, A.; Brown, S.; Kagoshima, S.; Kanoda, K.; Kuroki, K.; Mori, H.; Ogata, M.; Uji, S.; Wosnitza, J. Recent Topics of Organic Superconductors. J. Phys. Soc. Jpn. 2012, 81, 011004. [CrossRef]

10. Minamidate, T.; Oka, Y.; Shindo, H.; Yamazaki, T.; Matsunaga, N.; Nomura, K.; Kawamoto, A. Superconducting Phase in $\lambda$-(BEDT-STF $)_{2} \mathrm{GaCl}_{4}$ at High Pressures. J. Phys. Soc. Jpn. 2015, 84, 063704. [CrossRef]

11. Saito, Y.; Nakamura, H.; Sawada, M.; Yamazaki, T.; Fukuoka, S.; Matsunaga, N.; Nomura, K.; Dressel, M.; Kawamoto, A. Disordered quantum spin state in the stripe lattice system consisting of triangular and square tilings investigated by ${ }^{13} \mathrm{C} N M R$. arXiv 2019, arXiv:1910.09963.

12. Saito, Y.; Fukuoka, S.; Kobayashi, T.; Kawamoto, A.; Mori, H. Antiferromagnetic Ordering in Organic Conductor $\lambda$-(BEDTTTF $)_{2} \mathrm{GaCl}_{4}$ Probed by ${ }^{13} \mathrm{C}$ NMR. J. Phys. Soc. Jpn. 2018, 87, 013707. [CrossRef]

13. Hotta, C. Quantum electric dipoles in spin-liquid dimer Mott insulator $\kappa-\mathrm{ET}_{2} \mathrm{Cu}_{2}(\mathrm{CN})_{3}$. Phys. Rev. B 2010, 82, 241104. [CrossRef]

14. Hotta, C. Theories on Frustrated Electrons in Two-Dimensional Organic Solids. Crystals 2012, 2, 1155-1200. [CrossRef]

15. Dayal, S.; Clay, R.T.; Li, H.; Mazumdar, S. Paired electron crystal: Order from frustration in the quarter-filled band. Phys. Rev. $B$ 2011, 83, 245106. [CrossRef]

16. Clay, R.; Mazumdar, S. From charge- and spin-ordering to superconductivity in the organic charge-transfer solids. Phys. Rep. 2019, 788, 1-89. [CrossRef]

17. Lunkenheimer, P.; Müller, J.; Krohns, S.; Schrettle, F.; Loidl, A.; Hartmann, B.; Rommel, R.; De Souza, M.; Hotta, C.; Schlueter, J.A.; et al. Multiferroicity in an organic charge-transfer salt that is suggestive of electric-dipole-driven magnetism. Nat. Mater. 2012, 11, 755-758. [CrossRef]

18. Tomić, S.; Pinterić, M.; Ivek, T.; Sedlmeier, K.; Beyer, R.; Wu, D.; Schlueter, J.A.; Schweitzer, D.; Dressel, M. Magnetic ordering and charge dynamics in $\kappa-(\mathrm{BEDT}-\mathrm{TTF})_{2} \mathrm{Cu}\left[\mathrm{N}(\mathrm{CN})_{2}\right.$ ]Cl. J. Phys. Condens. Matter 2013, 25, 436004. [CrossRef] [PubMed]

19. Abdel-Jawad, M.; Terasaki, I.; Sasaki, T.; Yoneyama, N.; Kobayashi, N.; Uesu, Y.; Hotta, C. Anomalous dielectric response in the dimer Mott insulator $\kappa-(B E D T-T T F){ }_{2} \mathrm{Cu}_{2}(\mathrm{CN})_{3}$. Phys. Rev. B 2010, 82, 125119. [CrossRef]

20. Pinterić, M.; Čulo, M.; Milat, O.; Basletić, M.; Korin-Hamzić, B.; Tafra, E.; Hamzić, A.; Ivek, T.; Peterseim, T.; Miyagawa, K.; et al. Anisotropic charge dynamics in the quantum spin-liquid candidate $\kappa-(B E D T-T T F)_{2} \mathrm{Cu}_{2}(\mathrm{CN})_{3}$. Phys. Rev. B 2014, 90, 195139. [CrossRef]

21. Pinterić, M.; Lazić, P.; Pustogow, A.; Ivek, T.; Kuveždić, M.; Milat, O.; Gumhalter, B.; Basletić, M.; Čulo, M.; Korin-Hamzić, B.; et al. Anion effects on electronic structure and electrodynamic properties of the Mott insulator $\kappa-(\mathrm{BEDT}-\mathrm{TTF})_{2} \mathrm{Ag}{ }_{2}(\mathrm{CN})_{3}$. Phys. Rev. B 2016, 94, 161105. [CrossRef]

22. Pinterić, M.; Rivas Góngora, D.; Rapljenović, Ž.; Ivek, T.; Čulo, M.; Korin-Hamzić, B.; Milat, O.; Gumhalter, B.; Lazić, P.; Sanz Alonso, M.; et al. Electrodynamics in Organic Dimer Insulators Close to Mott Critical Point. Crystals 2018, 8, 190. [CrossRef]

23. Dressel, M.; Lazić, P.; Pustogow, A.; Zhukova, E.; Gorshunov, B.; Schlueter, J.A.; Milat, O.; Gumhalter, B.; Tomić, S. Lattice vibrations of the charge-transfer salt $\kappa-(\mathrm{BEDT}-\mathrm{TTF})_{2} \mathrm{Cu}_{2}(\mathrm{CN})_{3}$ : Comprehensive explanation of the electrodynamic response in a spin-liquid compound. Phys. Rev. B 2016, 93, 081201. [CrossRef]

24. Ivek, T.; Beyer, R.; Badalov, S.; Čulo, M.; Tomić, S.; Schlueter, J.A.; Zhilyaeva, E.I.; Lyubovskaya, R.N.; Dressel, M. Metal-insulator transition in the dimerized organic conductor $\kappa-(\mathrm{BEDT}-\mathrm{TTF})_{2} \mathrm{Hg}(\mathrm{SCN})_{2}$ Br. Phys. Rev. B 2017, 96, 085116. [CrossRef]

25. Nad, F.; Monceau, P Dielectric Response of the Charge Ordered State in Quasi-One-Dimensional Organic Conductors. J. Phys. Soc. Jpn. 2006, 75, 051005. [CrossRef]

26. Ishihara, S. Electronic ferroelectricity in molecular organic crystals. J. Phys. Condens. Matter 2014, 26, 493201. [CrossRef]

27. Monceau, P.; Nad, F.Y.; Brazovskii, S. Ferroelectric Mott-Hubbard Phase of Organic (TMTTF) ${ }_{2}$ X Conductors. Phys. Rev. Lett. 2001, 86, 4080-4083. [CrossRef]

28. Horiuchi, S.; Tokura, Y. Organic ferrolectrics. Nat. Mater. 2008, 7, 357-366. [CrossRef]

29. Mistewicz, K. Recent Advances in Ferroelectric Nanosensors: Toward Sensitive Detection of Gas, Mechanothermal Signals, and Radiation. J. Nanomater. 2018, 2018, 2651056. [CrossRef]

30. Zhang, S.; Malič, B.; Li, J.F.; Rödel, J. Lead-free ferroelectric materials: Prospective applications. J. Mater. Res. 2021, 36, 985-995. [CrossRef]

31. Asadi, K. (Ed.) Organic Ferroelectric Materials and Applications; Woodhead Publishing: Sawston, UK, 2021. 
32. Kim, T.Y.; Kim, S.K.; Kim, S.W. Application of ferroelectric materials for improving output power of energy harvesters. Nano Converg. 2018, 5, 30. [CrossRef]

33. Dressel, M.; Drichko, N. Optical Properties of Two-Dimensional Organic Conductors: Signatures of Charge Ordering and Correlation Effects. Chem. Rev. 2004, 104, 5689-5715. [CrossRef] [PubMed]

34. Drichko, N.; Kaiser, S.; Sun, Y.; Clauss, C.; Dressel, M.; Mori, H.; Schlueter, J.; Zhyliaeva, E.; Torunova, S.; Lyubovskaya, R. Evidence for charge order in organic superconductors obtained by vibrational spectroscopy. Phys. B 2009, 404, 490-493. [CrossRef]

35. Girlando, A. Charge Sensitive vibrations and electron-molecular vibration coupling in bis (ethylenedithio)-tetrathiafulvalene (BEDT-TTF). J. Phys. Chem. C 2011, 115, 19371. [CrossRef]

36. Girlando, A.; Masino, M.; Schlueter, J.; Drichko, N.; Kaiser, S.; Dressel, M. Spectroscopic characterization of charge order fluctuations in BEDT-TTF metals and superconductors. Phys. Stat. Sol. 2012, 249, 953-956. [CrossRef]

37. Yakushi, K. Infrared and Raman Studies of Charge Ordering in Organic Conductors, BEDT-TTF Salts with Quarter-Filled Bands. Crystals 2012, 2, 1291-1346. [CrossRef]

38. Girlando, A.; Masino, M.; Kaiser, S.; Sun, Y.; Drichko, N.; Dressel, M.; Mori, H. Charge-order fluctuations and superconductivity in two-dimensional organic metals. Phys. Rev. B 2014, 89, 174503. [CrossRef]

39. Mori, H.; Suzuki, H.; Okano, T.; Moriyama, H.; Nishio, Y.; Kajita, K.; Kodani, M.; Takimiya, K.; Otsubo, T. Positional order and disorder of symmetric and unsymmetric BEDT-STF salts. J. Solid State Chem. 2002, 168, 626. [CrossRef]

40. Kremer, F.; Schönhals, A. Broadband Dielectric Spectroscopy; Springer Science \& Business Media: Berlin/Heidelberg, Germany, 2002.

41. Yamamoto, T.; Uruichi, M.; Yamamoto, K.; Yakushi, K.; Kawamoto, A.; Taniguchi, H. Examination of the Charge-Sensitive Vibrational Modes in Bis(ethylenedithio)tetrathiafulvalene. J. Phys. Chem. B 2005, 109, 15226-15235. [CrossRef]

42. Painelli, A.; Girlando, A. Electron-molecular vibration (e-mv) coupling in charge-transfer compounds and its consequences on the optical spectra: A theoretical framework. J. Chem. Phys. 1986, 84, 5655. [CrossRef]

43. Iguchi, S.; Sasaki, S.; Yoneyama, N.; Taniguchi, H.; Nishizaki, T.; Sasaki, T. Relaxor ferroelectricity induced by electron correlations in a molecular dimer Mott insulator. Phys. Rev. B 2013, 87, 075107. [CrossRef]

44. Tomić, S.; Dressel, M. Ferroelectricity in molecular solids: A review of electrodynamic properties. Rep. Prog. Phys. 2015, 78, 096501. [CrossRef] [PubMed]

45. Jonscher, A.K. Dielectric Relaxation in Solids; Chelsea Dielectric Press: London, UK, 1983.

46. Jonscher, A.K. Dielectric relaxation in solids. J. Phys. D 1999, 32, R57. [CrossRef]

47. Lunkenheimer, P.; Loidl, A. Dielectric spectroscopy on organic charge-transfer salts. J. Phys. Condens. Matter 2015, 27, 373001. [CrossRef] [PubMed]

48. Cross, L.E. Relaxor ferroelectrics. Ferroelectrics 1987, 76, 241. [CrossRef]

49. Levstik, A.; Kutnjak, Z.; Filipič, C.; Pirc, R. Glassy freezing in relaxor ferroelectric lead magnesium niobate. Phys. Rev. B 1998, 57, 11204-11211. [CrossRef]

50. Hattori, Y.; Iguchi, S.; Sasaki, T.; Iwai, S.; Taniguchi, H.; Kishida, H. Electric-field-induced intradimer charge disproportionation in the dimer-Mott insulator $\beta^{\prime}-(\mathrm{BEDT}-\mathrm{TTF})_{2} \mathrm{ICl}_{2}$. Phys. Rev. B 2017, 95, 085149. [CrossRef]

51. Saito, Y.; Rösslhuber, R.; Löhle, A.; Sanz Alonso, M.; Wenzel, M.; Kawamoto, A.; Pustogow, A.; Dressel, M. Bandwidth-tuning from insulating Mott quantum spin liquid to Fermi liquid via chemical substitution in $\kappa-\left[(\mathrm{BEDT}-\mathrm{TTF})_{1-x}(\mathrm{BEDT}-\mathrm{STF})_{x}\right]_{2} \mathrm{Cu} \mathrm{u}_{2}(\mathrm{CN})_{3}$. arXiv 2021, arXiv:1911.06766. doi:10.1039/D1TC00785H.

52. Shinaoka, H.; Imada, M. Theory of Electron Transport near Anderson-Mott Transitions. J. Phys. Soc. Jpn. 2010, 79, 113703. [CrossRef]

53. Iakutkina, O.; Uykur, E.; Kobayashi, T.; Kawamoto, A.; Dressel, M.; Saito, Y. Charge imbalance in $\lambda$-(BETS) ${ }_{2} \mathrm{GaCl}_{4}$ and their interplay with superconductivity. Phys. Rev. B 2021, 104, 045108. [CrossRef]

54. Olejniczak, I.; Graja, A.; Kushch, N.D.; Cassoux, P.; Kobayashi, H. Polarized IR Reflectance Studies of the Organic Conductor $\kappa$-(BETS) ${ }_{2} \mathrm{FeCl}_{4}$. J. Phys. I Fr. 1996, 6, 1631-1641. [CrossRef]

55. Dressel, M.; Dumm, M.; Knoblauch, T.; Masino, M. Comprehensive Optical Investigations of Charge Order in Organic Chain Compounds (TMTTF) $)_{2}$ X. Crystals 2012, 2, 528-578. [CrossRef]

56. Beyer, R.; Dengl, A.; Peterseim, T.; Wackerow, S.; Ivek, T.; Pronin, A.V.; Schweitzer, D.; Dressel, M. Pressure-dependent optical investigations of $\alpha$-(BEDT-TTF $)_{2} \mathrm{I}_{3}$ : Tuning charge order and narrow gap towards a Dirac semimetal. Phys. Rev. B 2016, $93,195116$. [CrossRef]

57. Hiraki, K.; Kitahara, M.; Takahashi, T.; Mayaffre, H.; Horvatić, M.; Berthier, C.; Uji, S.; Tanaka, H.; Zhou, B.; Kobayashi, A.; et al. Evidence of Charge Disproportionation in $\lambda$ Type BETS Based Organic Superconductors. J. Phys. Soc. Jpn. 2010, $79,074711$. [CrossRef]

58. Sedlmeier, K.; Elsässer, S.; Neubauer, D.; Beyer, R.; Wu, D.; Ivek, T.; Tomić, S.; Schlueter, J.A.; Dressel, M. Absence of charge order in the dimerized $\kappa$-phase BEDT-TTF salts. Phys. Rev. B 2012, 86, 245103. [CrossRef]

59. Ivek, T.; Korin-Hamzić, B.; Milat, O.; Tomić, S.; Clauss, C.; Drichko, N.; Schweitzer, D.; Dressel, M. Collective Excitations in the Charge-Ordered Phase of $\alpha$-(BEDT-TTF $)_{2} \mathrm{I}_{3}$. Phys. Rev. Lett. 2010, 104, 206406. [CrossRef] [PubMed]

60. Ivek, T.; Korin-Hamzić, B.; Milat, O.; Tomić, S.; Clauss, C.; Drichko, N.; Schweitzer, D.; Dressel, M. Electrodynamic response of the charge ordering phase: Dielectric and optical studies of $\alpha$-(BEDT-TTF) ${ }_{2} \mathrm{I}_{3}$. Phys. Rev. B 2011, 83, 165128. [CrossRef] 
61. Yakushi, K.; Yamamoto, K.; Yamamoto, T.; Saito, Y.; Kawamoto, A. Raman spectroscopy study of charge fluctuation in the spin-liquid candidate $\kappa$-(BEDT-TTF $)_{2} \mathrm{Cu}_{2}(\mathrm{CN})_{3}$. J. Phys. Soc. Jpn. 2015, 84, 084711. [CrossRef]

62. Hassan, N.; Cunningham, S.; Mourigal, M.; Zhilyaeva, E.I.; Torunova, S.A.; Lyubovskaya, R.N.; Schlueter, J.A.; Drichko, N. Evidence for a quantum dipole liquid state in an organic quasi-two-dimensional material. Science 2018, 360, 1101-1104. [CrossRef]

63. Kobayashi, T.; Kawamoto, A. Evidence of antiferromagnetic fluctuation in the unconventional superconductor $\lambda$-(BETS $)_{2} \mathrm{GaCl}_{4}$ by ${ }^{13}$ C NMR. Phys. Rev. B 2017, 96, 125115. [CrossRef] 